\title{
Magnetic excitations in an ionic spin-chain system with a nonmagnetic ferroelectric instability
}

\author{
K. Sunami $\odot,{ }^{1, *}$ Y. Sakai, ${ }^{1}$ R. Takehara $\odot,{ }^{1}$ H. Adachi $\odot,{ }^{1}$ K. Miyagawa $\odot,{ }^{1}$ S. Horiuchi, ${ }^{2}$ and K. Kanoda $\odot^{1, \dagger}$ \\ ${ }^{1}$ Department of Applied Physics, University of Tokyo, Bunkyo-ku, Tokyo 113-8656, Japan \\ ${ }^{2}$ Research Institute for Advanced Electronics and Photonics (RIAEP), National Institute of Advanced Industrial Science and Technology \\ (AIST), Tsukuba, Ibaraki 305-8565, Japan
}

(Received 25 September 2020; revised 12 November 2020; accepted 13 November 2020; published 7 December 2020)

\begin{abstract}
Cross-correlation between magnetism and dielectric is expected to offer distinct emergent phenomena. Here, magnetic excitations in the organic donor-acceptor spin-chain system, tetrathiafulvalene-bromanil (TTF-BA), with a ferroelectric ground state is investigated by ${ }^{1} \mathrm{H}-\mathrm{NMR}$ spectroscopy. A nonmagnetic transition with a ferroelectric order is marked by sharp drops in the NMR shift and the nuclear spin relaxation rate $T_{1}^{-1}$ at $53 \mathrm{~K}$. Remarkably, the analyses of the NMR shift and $T_{1}^{-1}$ dictate that the paramagnetic spin susceptibility in TTF-BA is substantially suppressed from that expected for the one-dimensional Heisenberg spins. We propose that the spin-lattice coupling and the ferroelectric instability cooperate to promote precursory polar singlet formation in the ionic spin system with a nonmagnetic ferroelectric instability.
\end{abstract}

DOI: 10.1103/PhysRevResearch.2.043333

\section{INTRODUCTION}

One-dimensional (1D) systems possess coupled electronlattice instabilities, a metal-insulator (Peierls) transition for itinerant electron systems, and a paramagnetic-nonmagnetic (spin-Peierls) transition for localized spin systems [1,2]. Organic charge-transfer complexes of a quasi-1D nature are representative platforms for the study of these issues. Among them, tetrathiafulvalene-chloranil (TTF-CA) composed of 1D mixed stacks of donor molecules, TTF, and acceptor molecules, CA, is a fascinating material showing a neutralionic (NI) transition accompanied by a symmetry-breaking lattice dimerization at $81 \mathrm{~K}$ under ambient pressure [3,4]; it switches from a paraelectric neutral phase $\left(\mathrm{TTF}^{+\rho}-\mathrm{CA}^{-\rho}\right.$ with $\rho \sim 0.3)$ to a ferroelectric ionic phase $(\rho \sim 0.6-0.7)$. On the other hand, the analogous material, tetrathiafulvalenebromanil (TTF-BA), in which $\mathrm{Cl}$ atoms in CA molecules are substituted by $\mathrm{Br}$ atoms [Fig. 1(a)], is in a highly ionic state ( $\rho \sim 0.95)$ at all temperatures [5] with every molecule accommodating a $S=1 / 2$ spin, which is paramagnetic at room temperature. Upon cooling, TTF-BA exhibits a nonmagnetic dimerization transition with ferroelectricity [6,7], putatively a spin-Peierls transition, at $53 \mathrm{~K}$ [5].

Recent under-pressure studies of TTF-CA have revealed that the ionic phase above $\sim 9 \mathrm{kbar}$ is nondimerized and paraelectric around room temperature and undergoes a dimerized ferroelectric transition upon cooling [8,9], evoking a view that

\footnotetext{
*sunami@mdf2.t.u-tokyo.ac.jp

$\dagger$ kanoda@ap.t.u-tokyo.ac.jp

Published by the American Physical Society under the terms of the Creative Commons Attribution 4.0 International license. Further distribution of this work must maintain attribution to the author(s) and the published article's title, journal citation, and DOI.
}

TTF-BA is equivalent to TTF-CA under pressures above $\sim 9$ kbar. Notably, it has recently been shown that the magnetism and conductivity in the paraelectric ionic phase of TTF-CA under pressure are attributed to spin and charge solitons [10,11]. As shown in Fig. 1(b), however, the resistivity in TTF-BA (measured in the present study) is 6 to 7 orders of magnitude larger than that in TTF-CA under pressure [12], indicating the absence of charge-soliton excitations in TTFBA; thus, TTF-BA offers a distinct localized spin system with a polarized nonmagnetic ground state.

The present study aims to reveal the nature of spin excitations in TTF-BA, a polarizable Heisenberg antiferromagnetic spin-chain system with a nonmagnetic ferroelectric transition, by ${ }^{1} \mathrm{H}$-NMR measurements. In general, ${ }^{1} \mathrm{H}-\mathrm{NMR}$ has insufficient sensitivity for probing the electronic state because of small hyperfine coupling and molecular motions unwantedly contributing to the NMR relaxation rate. TTF-BA, however, has sizable ${ }^{1} \mathrm{H}$ hyperfine coupling constants, no motional molecular parts, and, further, appreciably large static and dynamical spin susceptibilities as described later. Owing to all these features of TTF-BA, ${ }^{1} \mathrm{H}-\mathrm{NMR}$ is competent for probing the spin states.

\section{EXPERIMENTAL}

We performed ${ }^{1} \mathrm{H}-\mathrm{NMR}$ measurements on a single crystal of TTF-BA under the magnetic field $H$ of $3.7 \mathrm{~T}$ applied parallel to the $b^{*}$ axis (perpendicular to the $a c$ plane). In this field configuration, there are four nonequivalent ${ }^{1} \mathrm{H}$ nuclei above $T_{\mathrm{c}}$ [denoted by $\mathrm{H}_{\alpha}(1)$ and $\mathrm{H}_{\alpha}(2)$ on the $\alpha$-stack running along the $a$ axis and $\mathrm{H}_{\beta}(1)$ and $\mathrm{H}_{\beta}(2)$ on the $\beta$ stack along the $b$ axis in Fig. 1(c)]. To acquire NMR signals, we employed the so-called solid-echo pulse sequence. The origin of the NMR line shift corresponds to the resonance frequency for tetramethylsilane (TMS). The spin-lattice relaxation rate $T_{1}^{-1}$ was determined by fitting the stretched 
(a)

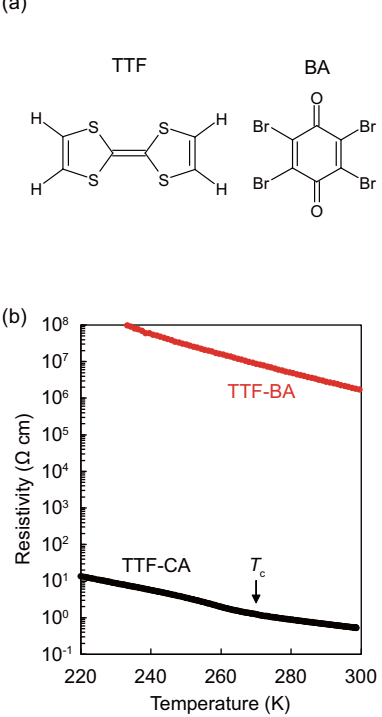

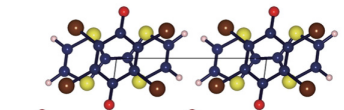
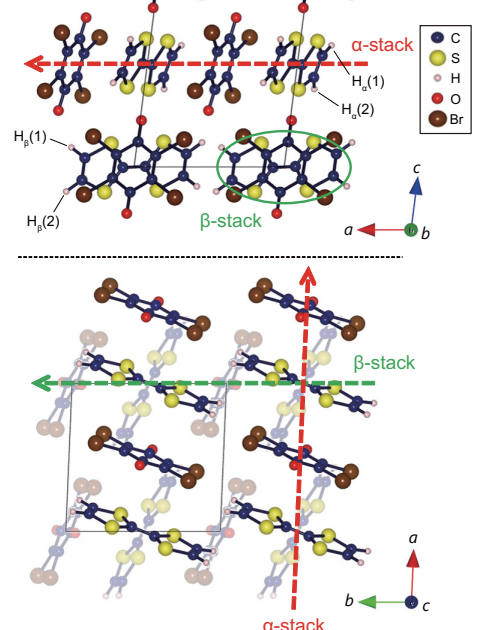

FIG. 1. (a) Molecular structures of TTF and BA. (b) Temperature dependence of electrical resistivity in TTF-BA under 5 kbar (red line) and TTF-CA under 14 kbar [12] (black line) measured by the four-terminal method. The resistance in TTF-BA at ambient pressure is too high $\left(>10^{6} \Omega\right)$ to measure by the four-terminal method, and thus we present the 5-kbar data. $T_{\mathrm{c}}$ is the ferroelectric transition temperature in TTF-CA at 14 kbar. (c) Crystal structures of TTF-BA viewed from the $b$ (upper) and $c$ (lower) axes [7].

exponential function to the relaxation curve of the nuclear magnetization obtained using the standard saturation method. The relaxation curve is nearly single exponential except below the nonmagnetic transition temperature, where a somewhat nonsingle exponential feature appears very probably due to the orphan spins failing to form singlets and/or minor impurity spins as seen later. The frequency dependence of $T_{1}^{-1}$ was measured in the range of $20-370 \mathrm{MHz}$, which corresponds to $0.5-8.6 \mathrm{~T}$.

\section{RESULTS}

\section{A. NMR spectra}

${ }^{1} \mathrm{H}-\mathrm{NMR}$ spectrum at around room temperature is formed by slightly asymmetric two broad peaks [Fig. 2(a)]. Upon cooling, the spectrum changes its shape but turns into two symmetric peaks below $\sim 50 \mathrm{~K}$. The spectral shift defined by the first moment of the entire spectrum is plotted in Fig. 2(b); it shows a maximum around $\sim 100 \mathrm{~K}$ and a sharp decrease indicating the nonmagnetic transition at $T_{\mathrm{c}}=53 \mathrm{~K}$. The spectral shift is contributed by the spin shift, proportional to the spin susceptibility $\chi$, and the temperature-independent chemical shift. These are separated by plotting the shift values against the previously reported $\chi$ values [6] [inset of Fig. 2(b)]; the slope of the linearity gives the hyperfine coupling component parallel to the field direction averaged for the four ${ }^{1} \mathrm{H}$ sites, $a_{\|}^{\text {ave }}$, of $-0.47 \mathrm{kOe} / \mu_{\mathrm{B}}$, and the intercept determines the chemical shift of $-37 \mathrm{ppm}$, which has an uncertainty of tens of ppm but is not involved in the present analysis.

The hyperfine coupling tensors have anisotropies arising from the dipolar interactions between nuclear and electron
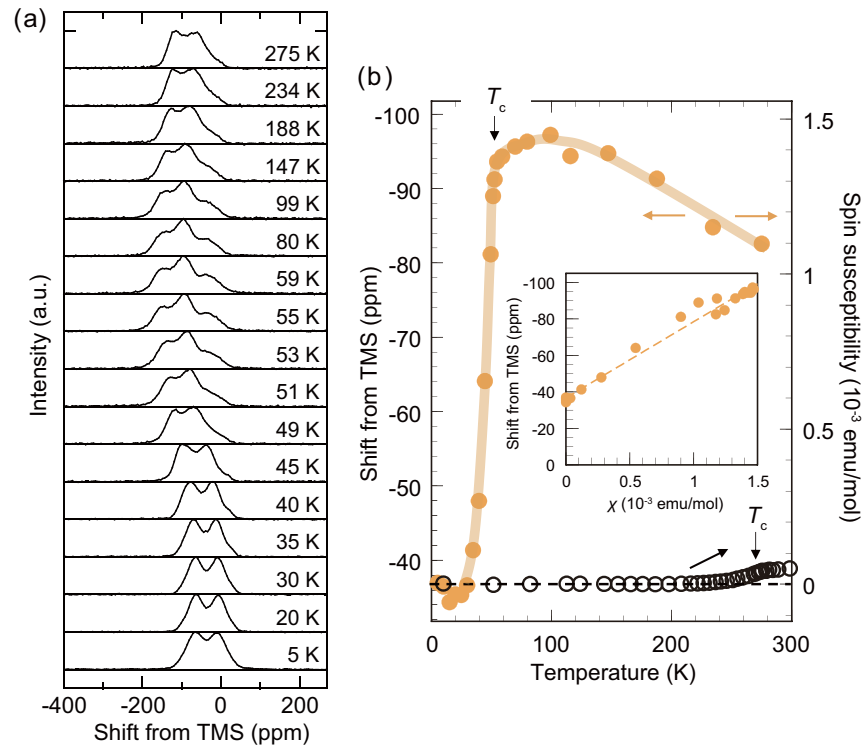

FIG. 2. Temperature profiles of the ${ }^{1} \mathrm{H}-\mathrm{NMR}$ spectra (a) and the first moments of spectra in TTF-BA (solid yellow circles) (b). In panel (b), the right axis denotes the scale of the spin susceptibility $\chi$ and the open black circles represent $\chi$ in TTF-CA under $14 \mathrm{kbar}$ [12]. Inset of panel (b): Plot of spectral shift vs spin susceptibility reported in Ref. [6] in TTF-BA.

spins. We evaluate a dipolar field at each ${ }^{1} \mathrm{H}$ site generated by the electron spin as follows: (i) a magnetic moment of $1 \mu_{\mathrm{B}}$ is distributed over the atomic sites (precisely, nuclear positions) in a molecule according to the Mulliken populations calculated by the extended Hückel method [13] using the atomic coordinates at $293 \mathrm{~K}$ reported in Ref. [7], and (ii) we incorporate dipole fields from electron spins on six neighboring molecules. For the present field configuration $\left(H \| b^{*}\right)$, the dipole hyperfine coupling components parallel to the field direction are calculated to be $0.35,0.41,-0.22$, and -0.32 $\mathrm{kOe} / \mu_{\mathrm{B}}$ for $\mathrm{H}_{\alpha}(1), \mathrm{H}_{\alpha}(2), \mathrm{H}_{\beta}(1)$, and $\mathrm{H}_{\beta}(2)$, respectively, whose average $\left(a_{\|}^{\text {aniso }}\right)^{\text {ave }}$ is $0.05 \mathrm{kOe} / \mu_{\mathrm{B}}$. The observed value of $-0.47 \mathrm{kOe} / \mu_{\mathrm{B}}$ is contributed by the dipole (anisotropic) part, $\left(a_{\|}^{\text {aniso }}\right)^{\text {ave }}$, and an isotropic part, $a^{\text {iso }}$, which thus yields $-0.52(=-0.47-0.05) \mathrm{kOe} / \mu_{\mathrm{B}}$; this value is a little different from $a^{\text {iso }}=-0.39 \mathrm{kOe} / \mu_{\mathrm{B}}$ evaluated in TTF-CuBDT [14]. The isotropic part $a^{\text {iso }}$ is determined by the contact interaction from the on-site electron spin at the ${ }^{1} \mathrm{H}$ site and the transferred hyperfine coupling from the electron spin at the adjacent carbon site; the former is negligibly small for the ${ }^{1} \mathrm{H}$ site located on the edges of molecule. For the latter, the spin density at the adjacent carbon site is affected by the surroundings such as the neighboring molecular species and their arrangements, and thus the size of $a^{\text {iso }}$ is likely to vary from material to material. The asymmetric shape of spectra above $T_{\mathrm{c}}$ possibly arises from the different anisotropic local fields at the four ${ }^{1} \mathrm{H}$ sites as estimated above. Below $T_{\mathrm{c}}$, where the spin shift vanishes, the spectra take the shape of the so-called Pake doublet derived from the nuclear dipolar interactions between $\mathrm{H}_{\alpha(\beta)}(1)$ and $\mathrm{H}_{\alpha(\beta)}(2)$, possibly broadened by the anisotropic chemical shift tensor giving site-dependent shifts. 

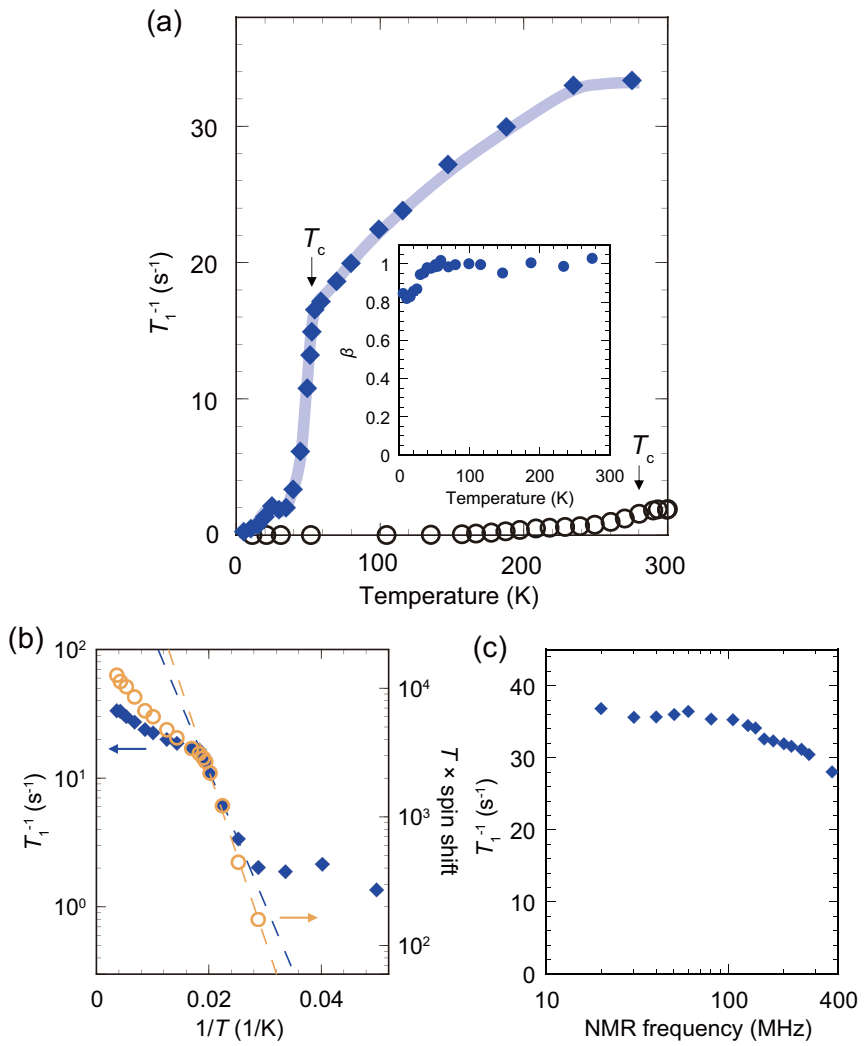

FIG. 3. (a) Temperature dependence of the ${ }^{1} \mathrm{H}-\mathrm{NMR}$ spin-lattice relaxation rate $T_{1}^{-1}$ in TTF-BA (solid blue diamonds) and TTF-CA under 13 kbar (open black circles) reported in Ref. [10]. Inset: Temperature dependence of the exponent $\beta$ in the stretched exponential fitting of the nuclear relaxation curves for TTF-BA. (b) Activation plots of $T_{1}^{-1}$ (solid blue diamonds) and spin shift multiplied by $T$ (open yellow circles) in TTF-BA. The broken lines indicate single exponential fits to the data in $35 \mathrm{~K}<T<T_{\mathrm{c}}$. (c) Frequency dependence of $T_{1}^{-1}$ at $280 \mathrm{~K}$ in TTF-BA.

\section{B. Spin-lattice relaxation rate $T_{1}^{-1}$}

The nonmagnetic transition was also captured by a steep decrease in the spin-lattice relaxation rate $T_{1}^{-1}$ below $T_{\mathrm{c}}$ [Fig. 3(a)]. The drop of $T_{1}^{-1}$ just below $T_{\mathrm{c}}$ is characterized by an activation energy $\Delta_{T_{1}^{-1}}$ of $240 \mathrm{~K}$, which is comparable with the activation value $\Delta_{s}, 300 \mathrm{~K}$, characterizing the drop in the spin shift multiplied by $T$ just below $T_{\mathrm{c}}$, as shown in Fig. 3(b). A leveling-off in $T_{1}^{-1}$ below $35 \mathrm{~K}$ is likely caused by free spins failing to form singlets and/or minor impurity spins, as often observed in ${ }^{1} \mathrm{H}-\mathrm{NMR}$ for spin-singlet phases [15]; the exponent in the stretched exponential fitting of the nuclear relaxation curve, which is nearly unity above $35 \mathrm{~K}$, decreases to 0.8 at $15 \mathrm{~K}$ [inset of Fig. 3(a)], also suggesting inhomogeneous nuclear relaxations by the dispersed orphan spins. The near agreement between the $\Delta_{T_{1}^{-1}}$ and $\Delta_{S}$ values is consistent with the singlet-triplet excitations unlike in the ferroelectric phase of TTF-CA showing a clear disagreement, which indicates that polaron excitations inheriting charge solitons vitally excited above $T_{\mathrm{c}}$ and singlet-triplet excitations occur in different energy scales and contribute to the shift and $T_{1}^{-1}$ with distinct weights [12]. The contrasting behaviors of TTF-CA and TTF-BA are reasonable because TTF-CA is situated near the NI phase boundary with the charge-transfer instability, whereas TTF-BA is an ionic Mott insulator with a large charge gap of $~ 8000 \mathrm{~K}$ [16], thus carrying low-energy excitations only in the spin degrees of freedom.

In the paramagnetic phase above $T_{\mathrm{c}}$, the spin susceptibility and $T_{1}^{-1}$ are much larger than those of TTF-CA under pressure [Figs. 2(b) and 3(a)], implying that TTF-BA is regarded as an $S=1 / 2$ localized spin system, not a soliton matter as in the ionic paraelectric phase of TTF-CA [10]. This picture is supported by the frequency-insensitive $T_{1}^{-1}$ observed at $280 \mathrm{~K}$ [Fig. 3(c)], which is compared to the pronounced frequency dependence of $T_{1}^{-1}$ pointing to the diffusive motion of spin solitons [10]. We note that, in 1D Heisenberg spin systems for the high-temperature limit, $T_{1}^{-1}$ is also expected to show the frequency dependence due to the classical spin diffusion [17,18]; thus, $T_{1}^{-1}$ insensitive to frequency implies that TTFBA cannot be regarded as the pure 1D spin system, which is discussed in more detail below, although the slight decrease of $T_{1}^{-1}$ at higher frequencies is possibly derived from the weak $1 \mathrm{D}$ nature of the spin chain.

\section{Evaluations of exchange interactions}

The lattice structure of TTF-BA is rather complicated as seen in Fig. 1(c), where the 1D chains running along the $a$ and $b$ axes stack alternatively along the $c$ direction with short TTF-TTF intercolumn contacts, quite different from TTF-CA where the 1D chains are arranged in parallel to each other [19]. We evaluated intermolecular transfer integrals in TTFBA through molecular orbital calculations [13]. The largest transfer integral is $t_{\|}=43-46 \mathrm{meV}$ between the TTF-HOMO (highest occupied molecular orbital) and the BA-LUMO (lowest unoccupied molecular orbital) along the $a$ or $b$ axis [the bold lines in Fig. 4(a)], the next largest one is $t_{\perp}=28 \mathrm{meV}$ between the TTF-HOMOs along the $c$ direction [the double lines in Fig. 4(a)], and the third largest one is $t_{\perp}^{\prime}=19 \mathrm{meV}$ between the TTF-HOMO in the $\alpha(\beta)$ chain and the CA-LUMO in the $\beta(\alpha)$ chain [the single lines in Fig. 4(a)]. Other values, for instance, the transfer integrals between the adjacent $\alpha(\beta)$ columns in parallel [the dotted lines in Fig. 4(a)], are below $\sim 10 \mathrm{meV}$.

For modeling the spin network of TTF-BA, we calculated the exchange interaction $J$ between the localized spins using the two expressions derived within the second-order perturbation in the ionic Hubbard model [20]: $J=2 t^{2} /(U-\Delta)+$ $2 t^{2} /(U+\Delta)$ for a donor-acceptor pair and $J=4 t^{2} / U$ for a donor-donor (acceptor-acceptor) pair, where $\Delta$ is the effective energy difference between the TTF-HOMO and BA-LUMO levels including the intersite Coulomb energy and $U$ is the onsite Coulomb energy. In this model, the charge transfer gap is expressed by $U-\Delta$, which is $0.8 \mathrm{eV}$ according to the infrared [5] and resistivity [16] measurements. With $U=1.5 \mathrm{eV}$ employed in the theoretical calculations [21], we obtained $\Delta=$ $0.7 \mathrm{eV}$, which yields $J_{\|} \equiv J_{\mathrm{TTF}(\alpha)-\mathrm{BA}(\alpha)}\left(\right.$ or $\left.J_{\mathrm{TTF}(\beta)-\mathrm{BA}(\beta)}\right)=$ $73-84 \mathrm{~K}, J_{\perp} \equiv J_{\mathrm{TTF}(\alpha)-\operatorname{TTF}(\beta)}=24 \mathrm{~K}$, and $J_{\perp}^{\prime} \equiv J_{\mathrm{TTF}(\alpha)-\mathrm{BA}(\beta)}$ $\left(\right.$ or $\left.J_{\mathrm{TTF}(\beta)-\mathrm{BA}(\alpha)}\right)=14 \mathrm{~K}$ [Fig. 4(a)]. Bewick and Soos computed $t_{\|}=60 \mathrm{meV}$ based on the semiempirical theory [22], which leads to $J_{\|}=140 \mathrm{~K}$, not far from the above calculation.

On the other hand, the exchange interactions can be evaluated from the experimental data of $T_{1}^{-1}$ using the following 


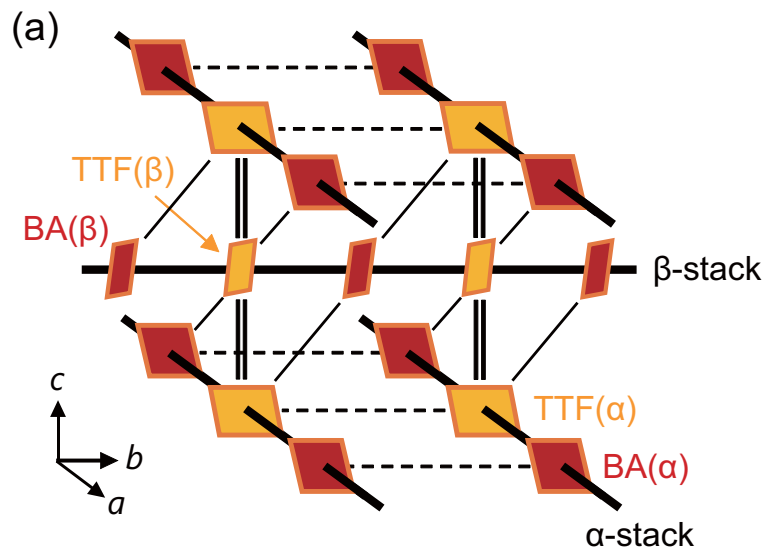

(b)

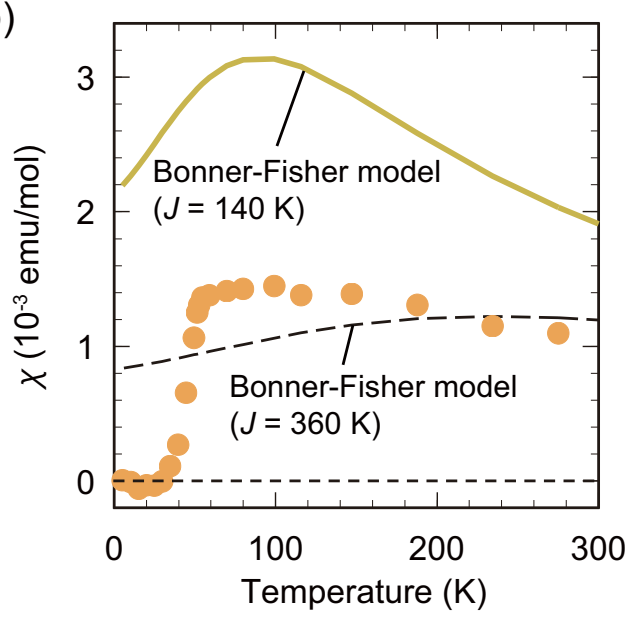

FIG. 4. (a) Network of transfer integrals (or the exchange interactions) in TTF-BA above $T_{\mathrm{c}}$. The bold, double, and single lines represent the nearest $\left(t_{\|}, J_{\|}\right)$, second-nearest $\left(t_{\perp}, J_{\perp}\right)$, and third-nearest $\left(t_{\perp}^{\prime}, J_{\perp}^{\prime}\right)$ neighboring molecular pairs, respectively. (b) Comparison between the experimental spin susceptibility and the Bonner-Fisher model calculations for $J=140 \mathrm{~K}$ (experimentally determined) and $J=360 \mathrm{~K}$ (for reference).

formula for localized spins in the high-temperature limit [23],

$$
T_{1}^{-1}=\sqrt{\frac{\pi S(S+1)}{3 Z}} \frac{g^{2} \hbar \gamma_{\mathrm{N}}^{2} a_{\perp}^{2}}{J},
$$

where $Z$ is the number of the neighboring sites, $g$ is the electron $g$-factor, $\gamma_{\mathrm{N}}$ is the nuclear gyromagnetic ratio, $\hbar$ is the reduced Planck constant, and $a_{\perp}$ is the hyperfine coupling component perpendicular to the field direction given by

$$
a_{\perp}^{2}=\frac{1}{2}\left[\left(a_{y y}^{2}+a_{z z}^{2}\right) H_{x}^{2}+\left(a_{z z}^{2}+a_{x x}^{2}\right) H_{y}^{2}+\left(a_{x x}^{2}+a_{y y}^{2}\right) H_{z}^{2}\right],
$$

where $a_{i i}(i=x, y$, and $z)$ is the principal value of the hyperfine coupling tensor and $H_{i}$ is the direction cosine of the applied field for the respective ${ }^{1} \mathrm{H}$ sites. In calculating $a_{\perp}$, we employed the $a^{\text {iso }}$ value of $-0.52 \mathrm{kOe} / \mu_{\mathrm{B}}$ as the isotropic part and calculated the anisotropic dipole part from the onmolecular spin, which yields $\left(-0.3 a^{\text {aniso }},-1.7 a^{\text {aniso }}, 2 a^{\text {aniso }}\right)$ with $a^{\text {aniso }} \sim 0.27 \mathrm{kOe} / \mu_{\mathrm{B}}$ in principal values. The principal axes of the hyperfine tensor differ among the four ${ }^{1} \mathrm{H}$ sites so that $H_{i}$ depends on the ${ }^{1} \mathrm{H}$ site. The average of $a_{\perp}^{2}$ over the four ${ }^{1} \mathrm{H}$ sites yields $\left(a_{\perp}^{2}\right)^{\text {ave }} \sim 0.44 \mathrm{kOe}^{2} / \mu_{\mathrm{B}}^{2}$ for the present field configuration of $H \| b^{*}$. Considering the interchain exchange interaction, we replace $J$ to $\sqrt{J_{\|}^{2}+J_{\perp}^{2}+J_{\perp}^{\prime 2}}$ in Eq. (1) with $J_{\|}=3-6 J_{\perp}=5-10 J_{\perp}^{\prime}$ and $Z=2$. The high-temperature limit of $T_{1}^{-1}$ is estimated at $41 \mathrm{~s}^{-1}$ by extrapolating it to $1 / T=0$ in Fig. 3(b). Substituting these values in Eq. (1), we obtained $J_{\|} \sim 140 \mathrm{~K}$, which is nearly in agreement with $J_{\|}=73-140 \mathrm{~K}$ calculated from the second-order perturbation formulas.

\section{DISCUSSION}

Anomalous spin excitations in TTF-BA are highlighted by comparing the spin susceptibilities derived from the spin shifts with the Bonner-Fisher curve expected in 1D antiferromagnetic (AF) Heisenberg spin systems [24,25] with $J=140 \mathrm{~K}$ obtained above. Bewick et al. calculated the spin susceptibility in the 1D mixed-stack charge-transfer systems described by the Peierls-Hubbard model [26], the temperature profile of which is close to the Bonner-Fisher curve for the ionic limit $(\rho \rightarrow 1)$. However, on the basis of the experimentally determined $J$ value, a large difference between the experimental data and the Bonner-Fisher curve is evident in TTF-BA with $\rho \sim 0.95$. We note that, for any $J$ value, the Bonner-Fisher curve does not reproduce the experimental susceptibility as reported in Refs. [5,6] and shown in Fig. 4(b) [27].

In general, the spin susceptibility is more and more reduced as the number of the neighboring sites increases, and thus the complicated spin network in TTF-BA appears responsible for the reduction of the susceptibility from that of the pure 1D spin chain. However, around temperatures of $\sim J_{\|}$and below, the intrachain AF correlations are developed so that, on a site in adjacent chains, the exchange fields produced through $J_{\perp}$ and $J_{\perp}^{\prime}$ [see Fig. 4(a)] are canceled by each other due to the frustration between $J_{\perp}$ and $J_{\perp}^{\prime}$. Thus, the spin network is expected to become 1D-like below $\sim 140 \mathrm{~K}$; then, the remarkable discrepancy between the experimental and calculated behaviors in TTF-BA invokes unusual mechanisms of spin excitations.

We discuss the origins of the discrepancy in the light of the distinctive nature of TTF-BA as a localized spin system. The first one is the enhanced spin-lattice coupling. Theoretically, working with the semiclassical treatment of a bosonized Hamiltonian for NI transition systems, Tsuchiizu et al. suggested that the spin susceptibility in the 1D AF Heisenberg spin systems with spin-lattice couplings and site-alternating potentials does not follow the Bonner-Fisher curve but carries unconventional excitations, called "spin polarons", unlike the conventional spinon excitations [28]. In particular, the spin-lattice coupling is expected to cause the local spin-singlet pairings even above $T_{\mathrm{c}}$ through the lattice fluctuations. Another exclusive feature of the present spin system is electric polarizability leading to polar fluctuations inherent in the mixed-stack ionic Mott insulator. Kagawa et al. reported that the conventional relationships $\Delta$ vs $T_{\mathrm{c}}$ and $H$ vs $T_{\mathrm{c}}$ in spin-Peierls systems are broken in TTF-BA [6]; typically, $\Delta / k_{\mathrm{B}} T_{\mathrm{c}}=2.5$ [29] and $\alpha=0.38$ [30] in the spin-Peierls systems, whereas $\Delta / k_{\mathrm{B}} T_{\mathrm{c}}=4.3$ and $\alpha=0.18$ in TTF-BA, where $\alpha$ is the coefficient given by 
$1-T_{\mathrm{c}}(H) / T_{\mathrm{c}}(0)=\alpha\left[g \mu_{\mathrm{B}} H / 2 k_{\mathrm{B}} T_{\mathrm{c}}(0)\right]^{2}$. The large $\Delta / k_{\mathrm{B}} T_{\mathrm{c}}$ value suggesting the spin gap far exceeding the energy scale of the transition and the small $\alpha$ value signifying outstanding robustness to magnetic field invoke an additional mechanism to stabilize the nonmagnetic phase beyond the conventional spin-Peierls framework. Polar fluctuations, which cause local donor-acceptor pairing, possibly favor precursory singlet formation in the paramagnetic phase, suppressing spin susceptibility above $T_{\mathrm{c}}$. The frequency-dependent dielectric constant above $T_{\mathrm{c}}$ reported in Ref. [16] is possibly related to the present scenario.

We note that the behavior of the spin susceptibility in TTF-BA resembles that of the inorganic spin-Peierls material $\mathrm{CuGeO}_{3}$ [31], in which its behavior can be quantitatively explained considering the next-nearest-neighbor (NNN) AF interaction in the spin chain [32]. In the present case of TTF-BA, however, the NNN interaction in the spin chain is vanishingly small due to the lack of the efficient path between the NNN sites, unlike the superexchange path in $\mathrm{CuGeO}_{3}$, and thus it is unlikely that the NNN interaction is the origin of the suppression of the spin susceptibility in TTF-BA.

It is a highly likely view that the precursory singlet fluctuations observed in TTF-BA manifest themselves in an extreme manner as a dimer liquid in TTF-CA, where most of the TTF and CA molecules form polar singlet pairs, whose long-range order is interrupted by soliton excitations. TTF-BA with a charge transfer of $\rho \sim 0.95$ is a nearly perfect ionic ferroelectric, whereas TTF-CA with $\rho \sim 0.6-0.7$ is an intermediately charge-transferred electronic ferroelectric. It is intriguing to see how the donor-acceptor spin chain system varies its magnetic excitations from the local spin regime to the soliton regime when $\rho$ is reduced from unity.

\section{CONCLUSION}

In conclusion, ${ }^{1} \mathrm{H}-\mathrm{NMR}$ spectroscopy of the alternating donor-acceptor ionic chain system TTF-BA revealed that it hosts an extraordinary spin system, understandable by neither the soliton matter realized in the analogous system TTF-CA nor the conventional Heisenberg spin system with the spinPeierls instability. The spin shift and the nuclear spin-lattice relaxation rate clearly captured the spin-singlet transition at $53 \mathrm{~K}$. The paramagnetic state above $53 \mathrm{~K}$ is demonstrated to host localized spin chains. However, the analyses of the spin shift and $T_{1}^{-1}$ found a substantial reduction in the spin susceptibility from that expected for the antiferromagnetic Heisenberg spin chains, evoking a view that TTF-BA offers an exclusive ionic spin system with unusually coupled magnetic and polar fluctuations enhanced prior to a nonmagnetic ferroelectric order. This potentially distinct cross-correlated fluctuation, which is possibly a generic feature for extensive ionic spin systems, is a profound addition to the correlated electron physics, awaiting theoretical challenges to treat jointly spin dynamics and electric polar correlation.

\section{ACKNOWLEDGMENTS}

This work was supported by the JSPS Grant-in-Aids for Scientific Research (Grants No. JP17K05532, No. JP18H05225, and No. JP19H01846) and by the Murata Science Foundation.
[1] R. E. Peierls, Quantum Theory of Solids (Clarendon, Oxford, 1955).

[2] M. C. Cross and D. S. Fisher, A new theory of the spinPeierls transition with special relevance to the experiments on TTFCuBDT, Phys. Rev. B 19, 402 (1979).

[3] J. B. Torrance, J. E. Vazquez, J. J. Mayerle, and V. Y. Lee, Discovery of a Neutral-to-Ionic Phase Transition in Organic Materials, Phys. Rev. Lett. 46, 253 (1981).

[4] J. B. Torrance, A. Girlando, J. J. Mayerle, J. I. Crowley, V. Y. Lee, P. Batail, and S. J. LaPlaca, Anomalous Nature of Neutralto-Ionic Phase Transition in Tetrathiafulvalene-Chloranil, Phys. Rev. Lett. 47, 1747 (1981).

[5] A. Girlando, C. Pecile, and J. B. Torrance, A key to understanding ionic mixed stacked organic solids: Tetrathiafulvalenebromanil (TTF-BA), Solid State Commun. 54, 753 (1985).

[6] F. Kagawa, S. Horiuchi, M. Tokunaga, J. Fujioka, and Y. Tokura, Ferroelectricity in a one-dimensional organic quantum magnet, Nat. Phys. 6, 169 (2010).

[7] P. García, S. Dahaoui, P. Fertey, E. Wenger, and C. Lecomte, Crystallographic investigation of temperature-induced phase transition of the tetrathiafulvalene- $p$-bromanil, TTF-BA charge transfer complex, Phys. Rev. B 72, 104115 (2005).

[8] M. Buron-Le Cointe, E. Collet, B. Toudic, P. Czarnecki, and H. Cailleau, Back to the structural and dynamical properties of neutral-ionic phase transitions, Crystals 7, 285 (2017).
[9] R. Takehara, K. Sunami, F. Iwase, M. Hosoda, K. Miyagawa, T. Miyamoto, H. Okamoto, and K. Kanoda, Revisited phase diagram on charge instability and lattice symmetry breaking in the organic ferroelectric TTF-QCl ${ }_{4}$, Phys. Rev. B 98, 054103 (2018).

[10] K. Sunami, T. Nishikawa, K. Miyagawa, S. Horiuchi, R. Kato, T. Miyamoto, H. Okamoto, and K. Kanoda, Evidence for solitonic spin excitations from a charge-lattice-coupled ferroelectric order, Sci. Adv. 4, eaau7725 (2018).

[11] R. Takehara, K. Sunami, K. Miyagawa, T. Miyamoto, H Okamoto, S. Horiuchi, R. Kato, and K. Kanoda, Topological charge transport by mobile dielectric-ferroelectric domain walls, Sci. Adv. 5, eaax8720 (2019).

[12] K. Sunami, R. Takehara, A. Katougi, K. Miyagawa, S. Horiuchi, R. Kato, T. Miyamoto, H. Okamoto, and K. Kanoda, Fate of a soliton matter upon symmetry-breaking ferroelectric order, arXiv:2008.09337.

[13] T. Mori, A. Kobayashi, Y. Sasaki, H. Kobayashi, G. Saito, and H. Inokuchi, The intermolecular interaction of tetrathiafulvalene and bis(ethylenedithio)tetrathiafulvalene in organic metals. Calculation of orbital overlaps and models of energy-band structures, Bull. Chem. Soc. Jpn. 57, 627 (1984).

[14] F. Devreux, C. Jeandey, M. Nechtschein, J. M. Fabre, and L. Giral, Electron-proton couplings and local susceptibilities in TTF and TCNQ salts, J. Phys. Fr. 40, 671 (1979). 
[15] T. Itou, A. Oyamada, S. Maegawa, K. Kubo, H. M. Yamamoto, and R. Kato, Superconductivity on the border of a spin-gapped Mott insulator: NMR studies of the quasi-two-dimensional organic system $\mathrm{EtMe}_{3} \mathrm{P}\left[\mathrm{Pd}(\mathrm{dmit})_{2}\right]_{2}$, Phys. Rev. B 79, 174517 (2009).

[16] Y. Tokura, S. Koshihara, Y. Iwasa, H. Okamoto, T. Komatsu, T. Koda, N. Iwasawa, and G. Saito, Domain-Wall Dynamics in Organic Charge-Transfer Compounds with OneDimensional Ferroelectricity, Phys. Rev. Lett. 63, 2405 (1989).

[17] J. Kikuchi, N. Kurata, K. Motoya, T. Yamauchi, and Y. Ueda, Spin diffusion in the $S=1 / 2$ quasi one-dimensional antiferromagnet $\alpha-\mathrm{VO}\left(\mathrm{PO}_{3}\right)_{2}$ via ${ }^{31} \mathrm{P}$ NMR, J. Phys. Soc. Jpn. 70, 2765 (2001).

[18] F. Borsa and M. Mali, Experimental study of high-temperature spin dynamics in one-dimensional Heisenberg paramagnets, Phys. Rev. B 9, 2215 (1974).

[19] J. J. Mayerle, J. B. Torrance, and J. I. Crowley, Mixed-stack complexes of tetrathiafulvalene. The structures of the chargetransfer complexes of TTF with chloranil and fluoranil, Acta. Crystallogr., Sect. B 35, 2988 (1979).

[20] H. Katsura, M. Sato, T. Furuta, and N. Nagaosa, Theory of the Optical Conductivity of Spin Liquid States in OneDimensional Mott Insulators, Phys. Rev. Lett. 103, 177402 (2009).

[21] N. Nagaosa and J. Takimoto, Theory of neutral-ionic transition in organic crystals. II. Effect of the intersite Coulomb interaction, J. Phys. Soc. Jpn. 55, 2745 (1986).

[22] S. A. Bewick and Z. G. Soos, Spin solitons in organic chargetransfer salts, Chem. Phys. 325, 60 (2006).
[23] T. Moriya, Nuclear magnetic relaxation in antiferromagnetics, Prog. Theor. Phys. 16, 23 (1956); Nuclear magnetic relaxation in antiferromagnetics, II, 16, 641 (1956).

[24] J. C. Bonner and M. E. Fisher, Linear magnetic chains with anisotropic coupling, Phys. Rev. 135, A640 (1964).

[25] W. E. Estes, D. P. Gavel, W. E. Hatfield, and D. J. Hodgson, Magnetic and structural characterization of dibromo- and dichlorobis(thiazole)copper(II), Inorg. Chem. 17, 1415 (1978).

[26] S. A. Bewick and Z. G. Soos, Peierls transitions in ionic organic charge-transfer crystals with spin and charge degrees of freedom, J. Phys. Chem. B 110, 18748 (2006).

[27] The definition of $J$ is different between Refs. [5,6] and the present paper. The former papers follow the paper by Bonner and Fisher (Ref. [24]), in which the Heisenberg Hamiltonian is described as $\mathcal{H}=2 J \Sigma \boldsymbol{S}_{i} \cdot \boldsymbol{S}_{j}$. In the present paper, we define $J=4 t^{2} / U$ and $\mathcal{H}=J \Sigma S_{i} \cdot S_{j}$ for the like-spin pair. Thus, the present $J$ value corresponds to twice the value in Refs. [5,6].

[28] M. Tsuchiizu, H. Yoshioka, and H. Seo, Phase competition, solitons, and domain walls in neutral-ionic transition systems, J. Phys. Soc. Jpn. 85, 104705 (2016).

[29] E. Orignac and R. Chitra, Mean-field theory of the spin-Peierls transition, Phys. Rev. B 70, 214436 (2004).

[30] M. C. Cross, Effect of magnetic fields on a spin-Peierls transition, Phys. Rev. B 20, 4606 (1979).

[31] M. Hase, I. Terasaki, and K. Uchinokura, Observation of the Spin-Peierls Transition in Linear $\mathrm{Cu}^{2+}($ Spin-1/2) Chains in an Inorganic Compound $\mathrm{CuGeO}_{3}$, Phys. Rev. Lett. 70, 3651 (1993).

[32] J. Riera and A. Dobry, Magnetic susceptibility in the spinPeierls system $\mathrm{CuGeO}_{3}$, Phys. Rev. B 51, 16098 (1995). 